\title{
THE LEFSCHETZ FIXED POINT THEOREM FOR NONCOMPACT LOCALLY CONNECTED SPACES
}

BY

\author{
R. J. KNILL
}

ABSTRACT. Leray's notion of convexoid space is localized and used to show that if $f: M \rightarrow M$ is a relatively compact map on a locally convex manifold $M$, and $f$ has no fixed points then its Lefschetz trace is zero. A similar theorem holds for certain adjunction spaces $Y \cup_{g} Z$ where $Y$ is $Q$-simplicial and $Z$ is locally convexoid. A number of other properties of locally convexoid spaces are derived; for example, any neighborhood retract of a locally convexoid space is locally convexoid.

The Lefschetz fixed point theorem has long been known to hold for compact manifolds, including compact homological manifolds. By restricting attention to compact maps, Leray extended the Lefschetz theorem to open subsets of Banach spaces and more recently, Browder and Eells have obtained it for infinite dimensional manifolds modelled on Banach and Fréchet spaces. The Leray, Browder and Eells results were each obtained by a reduction to the Lefschetz theorem for compact spaces. This same technique does not seem to apply to other infinite dimensional manifolds, such as those modelled on Montel spaces. In this paper we will consider this problem in the general context of regular Hausdorff locally connected spaces. For spaces which are locally connected in the sense of Čech, the Lefschetz fixed point theorem will be obtained for compact maps which have finite dimensional image. By localizing Leray's notion of convexoid space, and freeing it from compactness, it will be possible to obtain the Lefschetz theorem for compact maps of locally convex manifolds with no dimensional restrictions.

Because of its applicability to noncompact spaces, the notion of $Q$-simplicial space will be used throughout, along with related techniques. As an auxiliary to these techniques, a relative form of the classical method of acyclic carriers, the method of acyclic pairs of interlaced carriers, will be applied whenever it will be necessary to construct chain maps.

1. Preliminaries. With a few explicit changes, the conventions and notation of this paper are those of [16]; the most important are summarized here. All

Received by the editors June 30,1969 . $58 \mathrm{G} 10$.

AMS (MOS) subject classifications (1970). Primary 55C20; Secondary 57A20, 57B05,

Key words and phrases. Acyclic carrier, topological vector space, manifold, compact map, Lefschetz trace formula, $Q-s i m p l i c i a l$ space, cone, fixed point. 
spaces are regular Hausdorff. All homology and cohomology groups have for coefficient group a fixed field $Q$. Let $Y$ be a space and let $\alpha$ be a family of subsets of $Y$. For a subset $X$ of $Y, X_{a}$ is the abstract simplicial complex formed by the finite subsets of the sets $X \cap A$ where $A \in \alpha$, and $C_{*}\left(X_{\alpha}\right)$ is the oriented chain complex of $X_{a}$ with coefficients in the field $Q$. If $W$ is a subset of $X$, then $W_{\alpha}$ is a subcomplex of $X_{\alpha}$, and $C_{*}\left(W_{\alpha}\right) \subset C_{*}\left(X_{\alpha}\right)$. For a chain $c \in C_{*}\left(X_{\alpha}\right)$, the support sup $(c)$ is defined as

$$
\sup (c)=\bigcap\left\{W: c \in C_{*}\left(W_{\alpha}\right)\right\} .
$$

If $V$ is any subset of $Y$, then let the star of $\alpha$ on $V$ be the set

$$
\mathrm{St}_{\alpha}(V)=(\bigcup\{A \in \alpha: A \cap V \neq \varnothing\}) \cup V .
$$

Let $a^{*}=\left\{\right.$ St $\left._{\alpha}(A): A \in \alpha\right\}$.

For families $\alpha, \beta$ and $\gamma$ of subsets of $Y$, and a chain map $\phi: C_{*}\left(X_{\alpha}\right) \rightarrow$ $C_{*}\left(Y_{\beta}\right)$, one says that $\phi$ is subordinate to $\gamma$ if, for each chain $c$ in $C_{*}\left(X_{\alpha}\right)$,

$$
\mathrm{St}_{\gamma}(\sup (c)) \supset \sup \phi(c) \text {. }
$$

For example, if a refines $\beta, C_{*}\left(X_{\alpha}\right)$ is a subchain complex of $C_{*}\left(Y_{\beta}\right)$, and the inclusion is subordinate to any $\gamma$.

Generally $X$ will always be a subset of $Y$, and in this context an open covering of $X$ will mean a family $a$ of open subsets of $Y$ such that $\bigcup_{\alpha} \supset X$. The following definition of $Q$-simplicial ( $Q$ being the coefficient field, of course) is taken from [16]. $Y$ is $Q$-simplicial at a compact subset $X$ if for any open covering $\alpha$ of $X$ there exists an open covering $\alpha^{\#}=\alpha^{\#}(X)$ of $X$ with the property that for any open covering $\beta$ of $Y$, there is a chain map

$$
\omega: C_{*}\left(X_{a^{\sharp}}\right) \rightarrow C_{*}\left(Y_{\beta}\right)
$$

which is subordinate to $\alpha . \quad Y$ is $Q$-simplicial at a subset $Y^{\prime}$ if it is $Q$-simplicial at every compact subset of $Y^{\prime}$, and " $Y$ is $Q$-simplicial at $Y$," will be stated only as " $Y$ is $Q$-simplicial."

To anyone conversant with Lefschetz's quasi complex structures, the notion of $Q$-simplicial spaces is evidently a derivative of the former notion. The intent, as with quasicomplexes, is to impose conditions strong enough to be used in place of simplicial approximation to prove a type of Lefschetz fixed point theorem, but without imposing any extratopological structure in the fashion of semicomplex structures ([7], [25]), which are used for obtaining more information than we need.

If $Y$ and $Y^{\prime}$ are spaces and $f: Y \rightarrow Y^{\prime}$ is a map such that the induced Čech cohomology homomorphisms 


$$
H^{n}(f ; Q): H^{n}\left(Y^{\prime} ; Q\right) \rightarrow H^{n}(Y ; Q)
$$

are zero for all but finitely many values of $n$ and otherwise $H^{n}(f ; Q)$ has a finite dimensional image, then we say that $f$ has finite rank (over $Q$ ). If $X \subset Y^{\prime}$ is such that $f(Y) \subset X$, the corestriction of $f$ to $X$, is the map $f_{X}: Y \rightarrow X$ such that $f_{X}(y)=f(y)$ for all $y \in Y$.

In the case in which $Y=Y^{\prime}$, and $f$ has finite rank, one defines the Lefschetz number of $f$ in the usual fashion [21] as

$$
\Lambda(f ; Q)=\sum_{n \geq 0}(-1)^{n} \operatorname{tr} H^{n}(f ; Q) .
$$

The operational theorem of this paper is

1.1 Theorem [16]. Suppose that $Y$ is a space which is Q-simplicial at a compact subset $X$. Suppose further that $f: Y \rightarrow Y$ is a map such that $f(Y) \subset X$ and such that $f_{X}$ bas finite rank. If $\Lambda(f ; Q) \neq 0$, then $f$ has a fixed point.

A useful theorem which is deducible from the work of Dowker [10] is the following.

1.2 Theorem (Dowker). Suppose that $Y$ is a space, $X$ is a compact subspace and $a$ is an open covering of $X$. Then there is a finite subcomplex $K$ of $X_{a}$ and a chain map

$$
\pi: C_{*}\left(X_{\alpha}\right) \rightarrow C_{*}(K)
$$

which is subordinate to $a$. The dimension of $K$ may be assumed to be at most that of the nerve of $\alpha$.

2. The method of acyclic pairs of interlaced carriers. If $K$ is a simplicial complex and $C_{*}$ is an augmented chain complex over a field $Q$, then a carrier $\Gamma: K \rightarrow C_{*}$ is a function which assigns to each simplex $s$ of $K$ an augmented subchain complex $\Gamma(s)$ of $C_{*}$, subject to the condition that if $t$ is a face of $s$, then $\Gamma(t) \subset \Gamma(s)$. If $L$ is a subcomplex of $K$, a chain map $\omega: C_{*}(L) \rightarrow C_{*}$ is carried by $\Gamma$ if, for $s \in L, \omega C_{*}(\bar{s}) \subset \Gamma(s)$. (Here $\bar{s}$ is the subcomplex of $K$ formed by all faces of $s$.)

Definition. Suppose that $K$ is a simplicial complex, and $C_{*}$ is an augmented chain complex. A pair $\left(\Delta, \Delta^{\prime}\right)$, of carriers of $K$ into $C_{*}$ is said to be interlaced if, for a simplex $s$ of $K$, and proper face $t$ of $s$, we have $\Delta(t) \subset \Delta^{\prime}(s) \subset \Delta(s)$. For $p \geq 0$, a pair of interlaced carriers $\left(\Delta, \Delta^{\prime}\right)$ is $p$-acyclic if, for each integer $n, 0 \leq n \leq p$, and each $n$-simplex $s$ of $K$, the inclusion $\Delta^{\prime}(s) \rightarrow \Delta(s)$ induces the zero homomorphism of $\tilde{H}_{n}\left(\Delta^{\prime}(s)\right)$ into $\tilde{H}_{n}(\Delta(s))$, where $\tilde{H}_{n}$ is the $n$th reduced 
homology functor. If $\left(\Delta, \Delta^{\prime}\right)$ is $p$-acyclic for all $p \geq 0$, it is acyclic.

Example. Suppose that $K$ is a simplicial complex and $\Gamma^{0} \subset \Gamma^{1} \subset \cdots \subset \Gamma^{p+1}=$ $\Gamma$ is a filtration of carriers of $K$ into an augmented chain complex $C_{*}$. Then one defines a pair of interlaced carriers $\left(\Delta, \Delta^{\prime}\right)$ of $K$ into $C_{*}$, by letting, for an $n$-simplex $s$ of $K$,

$$
\Delta(s)=\Delta^{\prime}(s)=\Gamma(s) \text { if } n>p
$$

and let

$$
\Delta^{\prime}(s)=\Gamma^{n}(s), \quad \Delta(s)=\Gamma^{n+1}(s) \text { for } 0 \leq n \leq p .
$$

Then $\left(\Delta, \Delta^{\prime}\right)$ is $p$-acyclic if the inclusion $\Gamma^{n}(s) \rightarrow \Gamma^{n+1}(s)$ induces the zero homomorphism $\tilde{H}_{n}\left(\Gamma^{n}(s)\right) \rightarrow \tilde{H}_{n}\left(\Gamma^{n+1}(s)\right)$, for any $n$-simplex $s$ of $K$ and for any $0 \leq n \leq p$. If, in addition, $\operatorname{dim} K=p$, then $\left(\Delta, \Delta^{\prime}\right)$ is $\infty$-acyclic.

If $K$ is a simplicial complex, then $K^{p}$ denotes the $p$-dimensional skeleton of $K$,

$$
K^{p}=\{s \in K: \text { dimension } s \leq p\}
$$

We have the following easy generalization of the classical acyclic carrier theorem.

Theorem. If $\left(\Delta, \Delta^{\prime}\right)$ is a p-acyclic pair of interlaced carriers of a simplicial complex $K$ into an augmented chain complex $C_{*}$, and $L$ is a subcomplex of $K$, then

2.1. For any chain map $\omega: C_{*}(L) \rightarrow C_{*}$ which is carried by $\Delta$, there is an extension of $\omega$ to a chain map $\omega^{\prime}: C_{*}\left(L \cup K^{p+1}\right) \rightarrow C_{*}$ which is carried by $\Delta$.

2.2. For any two chain maps $\omega^{\prime}, \omega^{\prime \prime}: C_{*}\left(L \cup K^{p}\right) \rightarrow C_{*}$ which are carried by $\Delta^{\prime}$, and for any chain bomotopy $D: C_{*}(L) \rightarrow C_{*}$ between $\omega^{0} \mid C_{*}(L)$ and $\omega^{\prime \prime} \mid C_{*}(L)$ which is carried by $\Delta$, there is an extension of $D$ to a chain bomotopy $D^{\prime}: C_{*}\left(L \cup K^{p}\right) \rightarrow C_{*}$ of $\omega^{\prime}$ to $\omega^{\prime \prime}$, and which is carried by $\Delta$.

The proof is that of the classical acyclic carrier theorem, with appropriate modifications.

3. Local connectivity. In this section we discuss homological local connectedness in two forms. The first is a modification of Čech's (homological) local connectivity; the second is a localization of Leray's convexoid condition [20].

Suppose that $X$ is a subspace of a space $Y$, and $p$ is a nonnegative integer. $X$ is pacyclic in $Y$ if, for any open covering $a$ of $Y$, there is an open covering $\alpha^{\prime}$ of $X$ such that $\alpha^{\prime}>\alpha$ and the inclusion $X_{\alpha^{\prime}} \subset Y_{\alpha}$ induces zero reduced homology homomorphisms

$$
\tilde{H}_{n}\left(X_{a^{\prime}} ; Q\right) \rightarrow \tilde{H}_{n}\left(Y_{a} ; Q\right), \quad 0 \leq n \leq p .
$$


In case $X=Y$, we say that $Y$ is pacyclic. If $Y$ is pacyclic for all integers $p>0$, then $Y$ is $\infty$-acyclic.

This notion of $p$-acyclicity of $Y$ is stronger than the notion that $Y$ has the Čech homology groups of a point in all dimensions from 0 through $p$. The following theorem is well known.

3.1 Theorem. Suppose $Y$ is a space and $X$ is a compact subspace. Then $X$ is p-acyclic in $Y$ if and only if the inclusion $X \subset Y$ induces zero reduced Čech homology homomorphisms in all dimensions from 0 through $p$.

Definition. Let $Y$ be a regular Hausdorff space. For a point $y \in Y$ and integer $p>0, Y$ is " $\mathrm{lc}_{p}(Q)$ at $y$ " if, for any neighborhood $U$ of $y$, there exists a neighborhood $V$ of $y$ in $U$ such that $V$ is p-acyclic in $U . Y$ is $\operatorname{lc}_{p}(Q)$ at a subset $Y^{\prime}$ if it is $\operatorname{lc}_{p}(Q)$ at every point of $Y^{\prime}$.

In order to localize Leray's notion of convexoid spaces, we need to introduce the notion of monotone set function. A monotone set function $F$ on a space $Y$ is a function which assigns to each subset $W$ of $Y$ a set $F(W)$ such that two conditions hold:

(a) $W \subset F(W) \subset Y$,

(b) if $V \subset W$ then $F(V) \subset F(W)$.

If $F^{\prime}$ is a monotone set function, then $F$ is subordinate to $F^{\prime}$ if for each $W \subset Y, F(W) \subset F^{\prime}(W)$.

Note that, for each family $\alpha$ of subsets of $Y, \mathrm{St}_{\alpha}$ is a monotone set function.

For a family $a$ of subsets of a space $Y$ and subset $W$ of $Y$, write $\operatorname{diam}(W)<\alpha$ if, for some $A \in \alpha, W$ is a subset of $A$. Then $a$ is a gauge for $F$ if $F(W)$ is $\infty$-acyclic whenever $\operatorname{diam}(W)<a$.

Definition. Let $Y$ be a regular Hausdorff space. For compact $X \subset Y, Y$ is "locally convexoid at $X$ " if, for any open covering $a$ of $X$, there exists a monotone set function $F$ on $Y$ which is subordinate to St $\alpha$, and there exists an open covering $a^{\prime}$ of $X$ which is a gauge for $F$. We will say $Y$ is locally convexoid at a subset $Y^{\prime}$ if it is locally convexoid at every compact subset of $Y^{\prime}$; if $Y=Y^{\prime}$, we will simply say $Y$ is locally convexoid.

This definition is analogous to the previously given definition of $\mathrm{lc}_{p}(Q)$; however, a compact subset $X$ is used in place of a point $y$, an open covering $\alpha$ in place of a neighborhood $U$, and a monotone set function $F$ in place of a neighborhood $V$. The next two theorems justify the use of the terms "convexoid" and "locally."

3.2 Theorem. Suppose that $X$ is a compact convexoid space. Then $X$ is locally convexoid. 
Proof. Let $\alpha$ be an open covering of $X$. Then there is an open covering $\beta$ of $X$ such that $\beta^{*}>a$. From the definition of convexoid [20], there is a finite closed covering $\gamma$ of $X$ which refines $\beta$ and which has the property that for any

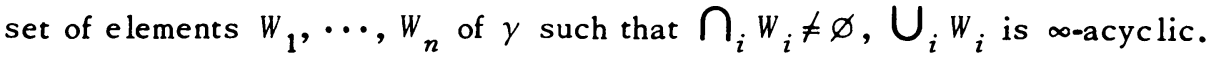
Then $F=\mathrm{St}_{\gamma}$ is a monotone set function which is subordinate to $\mathrm{St}_{\alpha}$. Define $\alpha^{\prime}$ as the family of all open sets of the form $X \backslash \bigcup\{W \in \gamma: W \not x\}$, where $x$ ranges over the points of $X$. Then $\alpha^{\prime}$ is a gauge for $F$ at $X$. Thus $X$ is locally convexoid.

3.3 Theorem. If $Y^{\prime}$ is a subset of $Y$, then any of the following is equivalent to $Y$ being locally convexoid at $Y^{\prime}$.

3.3.1. Some neighborbood $U$ of $Y^{\prime}$ is locally convexoid at $Y^{\prime}$.

3.3.2. Every neighborbood $U$ of $Y^{\prime}$ is locally convexoid at $Y^{\prime}$.

3.3.3. There are relatively open subsets $U_{i}$ of $Y^{\prime}$ such that $\bigcup_{i} U_{i}=Y^{\prime}$ and such that $Y$ is locally convexoid at each $U_{i}$.

Proof. If $Y$ is locally convexoid at $Y^{\prime}$ then one proves that 3.3 .2 holds as follows. For an $X$ compact, $X \subset Y^{\prime}$, and open covering $a$ of $X$ by subsets of $U$, there is a monotone set function $F$ on $Y$ which is subordinate to $a$, and open covering $a^{\prime}$ of $X, \alpha^{\prime}$ a gauge for $F$. Then the restriction of $F$ to the subsets of $U$ defines a monotone set function on $U$ subordinate to $\mathrm{St}_{a}$, with gauge $\left\{A \cap U: A \in a^{\prime}\right\}$.

Clearly 3.3.2 implies 3.3.1, and if 3.3.1 holds, then one may extend any monotone set function $F$ on $U$ to a monotone set function $F^{\prime}$ on $Y$ by letting $F^{\prime}(W)=F(W \cap U) \cup W$ for $W \in Y$. Then $F^{\prime}$ is subordinate to any St $a$ that $F$ is subordinate to, and any gauge for $F^{\prime}$ is a gauge for $F$, so if $U$ is locally convexoid at $Y^{\prime}$, so is $Y$.

Evidently, if $Y$ is locally convexoid then 3.3 .3 holds by letting $U_{i}=Y^{\prime}$ for all $i$. Conversely if 3.3 .3 holds, and $X$ is a compact subset of $Y^{\prime}$, then $X$ is a union of compact subsets $X=X_{1} \cup X_{2} \cup \cdots \cup X_{n}$ such that each $X_{j}$ is a subset of some $U_{i(j)^{*}}$. Thus, $Y$ is locally convexoid at each $X_{j}$. To see that $Y$ is locally convexoid at $X$, it suffices to prove the case in which $X=X_{1} \cup X_{2}$.

Let $\alpha$ be an open covering of $X$; we need to find a monotone set function $F$ on $Y$ which is subordinate to St ${ }_{a}$, and an open covering of $X$ which is a gauge for $F$. Let $\alpha_{1}$ be an open covering of $X$ such that $a_{1}^{*}>a$. There exist a monotone set function $F^{\prime}$ on $Y$ which is subordinate to $\mathrm{St}_{\alpha_{1}}$, and an open covering $a^{\prime}$ of $X_{1}$ which is a gauge for $F^{\prime}$. Assume that $a^{\prime}>a_{1}$. Let $\alpha_{2}$ be an open covering of $X_{2}$ such that $\alpha_{2}^{*}>a^{\prime} \cup\left\{A \backslash X_{1}: A \in a_{1}\right\}$. Then there is a monotone set function $F^{\prime \prime}$ on $Y$ which is subordinate to St $a_{2}$ and which has a gauge $\alpha^{n}$, where $\alpha^{\prime \prime}$ is an open covering of $X_{2^{*}}$. Assume that $a^{n}$ refines $a_{2}$. 
Let $\beta$ be an open covering of $X$ such that every set of $\beta$ meets $X$ and such that $\beta^{*}>\left\{A \backslash X_{2}: A \in \alpha^{\prime}\right\} \cup \alpha^{\prime \prime}$. Then define a monotone set function $F$ on $Y$ as follows. For a subset $W$ of $Y$, let

$$
F(W)= \begin{cases}F^{\prime}\left(F^{\prime \prime}\left(\mathrm{St}_{\beta}(W)\right)\right) & \text { if } \mathrm{St}_{\beta}(W) \cap X_{i} \neq \varnothing \text { for } i=1,2, \\ F^{\prime \prime}\left(\mathrm{St}_{\beta}(W)\right) & \text { if } \mathrm{St}_{\beta}(W) \cap X_{2} \neq \varnothing, \mathrm{St}_{\beta}(W) \cap X_{1}=\varnothing, \\ F^{\prime}\left(\mathrm{St}_{\beta}(W)\right) & \text { if } \mathrm{St}_{\beta}(W) \cap X_{1} \neq \varnothing, \mathrm{St}_{\beta}(W) \cap X_{2}=\varnothing, \\ \mathrm{St}_{\beta}(W) & \text { if } \mathrm{St}_{\beta}(W) \cap X_{i}=\varnothing \text { for } i=1,2 .\end{cases}
$$

Then one readily sees that $F$ is a monotone set function on $Y$. It is subordinate to $\mathrm{St}_{a}$ since, for any subset $W$ of $Y$,

$$
F(W) \subset F^{\prime}\left(F^{\prime \prime}\left(\mathrm{St}_{\beta}(W)\right)\right) \subset \mathrm{St}_{\alpha_{1}} \mathrm{St}_{a_{2}} \mathrm{St}_{\beta}(W) \subset \mathrm{St}_{\alpha_{1}} \mathrm{St}_{a_{1}}(W) \subset \mathrm{St}_{\alpha}(W) .
$$

Furthermore $\beta$ is a gauge for $F$ : If $W \subset B \in \beta$, then $\mathrm{St}_{\beta}(W)$ intersects $X$, and $\operatorname{diam~St}{ }_{\beta}(W)<\left\{A \backslash X_{2}: A \in \alpha^{\prime}\right\} \cup \alpha^{\prime \prime}$. There are three cases:

(1) If St ${ }_{\beta}(W)$ intersects $X_{1}$ and $X_{2}$, then diam St ${ }_{\beta}(W)<\alpha^{n}<\alpha_{2}$. But then $\operatorname{diam} F^{\prime \prime}\left(\mathrm{St}_{\beta}(W)\right)<\alpha^{\prime} \cup\left\{A \backslash X_{1}: A \in \alpha_{1}\right\}$. Since St ${ }_{\beta}(W) \cap X_{1} \neq \varnothing$, then $\operatorname{diam} F^{\prime \prime}\left(\mathrm{St}_{\beta}(W)\right)<\alpha^{\prime}$ so $F(W)=F^{\prime}\left(F^{\prime \prime}\left(\mathrm{St}_{\beta}(W)\right)\right)$ is $\infty$-acyclic.

(2) If $\mathrm{St}_{\beta}(W)$ intersects $X_{2}$ but not $X_{1}$, then $\operatorname{diam~St}{ }_{\beta}(W)<\alpha^{n}$ so $F(W)=$ $F^{\prime \prime}\left(\mathrm{St}_{\beta}(W)\right)$ is $\infty$-acyclic.

(3) If St ${ }_{\beta}(W)$ intersects $X_{1}$ but not $X_{2}$, then $\operatorname{diam~St}{ }_{\beta}(W)<\alpha^{\prime}$, so $F(W)=F^{\prime}\left(\mathrm{St}_{\beta}(W)\right)$ is $\infty$-acyclic.

Since $F$ has a gauge $\beta$, this completes the proof.

3.4 Theorem. Suppose that $Y$ is a space which is locally convexoid at a compact subset $X$. Then there is a neighborbood $U$ of $X$ such that the inclusion map $U \subset Y$ bas finite rank.

Proof. For this it suffices to find $U$ and a finite open covering $\beta$ of $U$ such that for $n \geq 0$ there is a cofinal family of coverings $\gamma$ of $Y$ for which there are homomorphisms

$$
H^{n}\left(Y_{\gamma} ; Q\right) \rightarrow H^{n}\left(U_{\beta} ; Q\right) \rightarrow H^{n}\left(U_{\gamma} ; Q\right)
$$

such that their composition is induced by the inclusion of $U_{\gamma}$ into $Y_{\gamma}$. There exist a monotone set function $F$ on $Y$ and an open covering $\alpha$ of $X$ which is a gauge for $F$. Let $\beta$ be a finite open covering of $X$ such that every set of $\beta$ intersects $X$, and such that $\beta^{*}>\alpha$. Let $U$ be a closed neighborhood of $X$ which is a subset of St $\beta(X)$. Let $n$ be a nonnegative integer. For any simplex $s \in U_{\beta}, F\left(\operatorname{St}_{\beta}(s)\right)$ is $\infty$ acyclic, since $\beta^{*}>\alpha$. So if $\gamma$ is any open covering of $Y$ which refines $\beta \cup\{Y \backslash U\}=\beta^{\prime}$, then there is a sequence of open coverings of $U$, 


$$
\gamma(-1)>\gamma(0)>\cdots>\gamma(n+1)>\gamma(n+2)=\gamma(n+3)=\cdots=\gamma
$$

such that, for $0 \leq i \leq n+2,\left[F\left(\mathrm{St}_{\beta}(s)\right)\right]_{\gamma(i-1)}$ s s a-acyclic in $\left[F\left(\mathrm{St}_{\beta}(s)\right)\right]_{\dot{\gamma}(i)}$. Since $\beta$ is finite, the family of sets of the form $F\left(\mathrm{St}_{\beta}(s)\right), s \in U_{\gamma}$, is finite. Thus the sequence of coverings $\{\gamma(i)\}_{i}$ may be chosen sa that $\left[F\left(\mathrm{St}_{\beta}(s)\right)\right]_{\gamma(i-1)}$ is $\infty$-acyclic in $\left[F\left(\mathrm{St}_{\beta}(s)\right)\right]_{\gamma(i)}$ for all $i, 0 \leq i \leq n+2$, and all simplexes $s \in U_{\beta}$. Now define three carriers $\Delta, \Delta^{\prime}, \Delta^{\prime \prime}: U_{\beta} \rightarrow C_{*}\left(U_{\gamma}\right)$ as follows. For any $m$-simplex $s$ of $U_{\gamma}$ let

$$
\begin{aligned}
\Delta(s) & =C_{*}\left(\left[F\left(\mathrm{St}_{\beta}(s)\right)\right]_{\gamma(m+1)}\right), \\
\Delta^{\prime}(s) & =C_{*}\left(\left[F\left(\mathrm{St}_{\beta}(s)\right)\right]_{\gamma(m)}\right), \\
\Delta^{\prime \prime}(s) & =C_{*}\left(\left[F\left(\mathrm{St}_{\beta}(s)\right)\right]_{\gamma(m-1)}\right) .
\end{aligned}
$$

Then $\left(\Delta, \Delta^{\prime}\right)$ and $\left(\Delta^{\prime}, \Delta^{\prime \prime}\right)$ are both $(n+1)$-acyclic pairs of interlaced carriers. For any simplicial complex $K$ contained in $U_{\beta}$ of dimension at most $n+1$, Theorem 2.1 applied to the pair $\left(\Delta^{\prime}, \Delta^{\prime \prime}\right)$ yields a chain map carried by $\Delta^{\prime}$,

(1) $\omega: C_{*}(K) \rightarrow C_{*}\left(U_{\gamma}\right)$,

and Theorem 2.2 applied to the pair $\left(\Delta, \Delta^{\prime}\right)$ shows

(2) $\omega$ is unique to within chain homotopy.

Now apply (1) to $K=(n+1)$-skeleton of $U_{\beta}$ to obtain $\omega$, and after passing to cohomology, a homomorphism $\omega^{n}: H^{n}\left(Y_{\gamma} ; Q\right) \rightarrow H^{n}\left(U_{\beta}, Q\right)$. Let

$$
\pi^{n}: H^{n}\left(U_{\beta} ; Q\right) \rightarrow H^{n}\left(U_{\gamma} ; Q\right)
$$

be the homomorphism induced by the inclusion $\pi: U_{\gamma} \subset U_{\beta}$. Now apply (2) to $K=(n+1)$-skeleton of $U_{\gamma}$ to obtain that the composition $\pi^{n} \omega^{n}$ is the homomorphism induced by the inclusion of $U_{\gamma}$ into $Y_{\gamma}$. As commented in the beginning of this proof, this proves 3.4 .

4. Fixed point theorems: The finite dimensional case. In this section we extend the Lefschetz fixed point theorem to locally compact cohomological manifolds, as well as to certain locally connected spaces without finitely generated homology groups. In most cases the form of the Lefschetz theorem to be obtained is that of Theorem 1.1 , in which case all that will be stated is that the spaces involved are $Q$-simplicial.

4.1 Theorem. Suppose that $p$ is a nonnegative integer, and $Y$ is a space which is $\mathrm{lc}_{p}(Q)$ at a compact subset $X$. If the covering dimension of $X$ is at most $p+1$, then $Y$ is $Q$-simplicial at $X$.

Proof. Let $\alpha$ be an open covering of $X$. Since $Y$ is $\mathrm{lc}_{p}(Q)$ at $X$, there is a sequence of finite open coverings of $X$, 


$$
\alpha(-1)>\alpha(0)>\cdots>\alpha(p+1)>\alpha(p+1)^{*}>\alpha
$$

with the property that if $B$ is a nonempty subset of $Y$, and $\operatorname{diam}(B)<\alpha(i-1)$, then $\mathrm{St}_{a(i-1)}(B)$ is $p$-acyclic in $\mathrm{St}_{a(i)}(B)$. We may assume that the dimension of the nerve of $\alpha(-1)$ is at most $p+1$. Let $\alpha^{\#}=\alpha(-1)$. We claim that for any open covering $\beta$ of $Y$ there is a chain map of $C_{*}\left(X_{\text {a\# }}\right)$ into $C_{*}\left(Y_{\beta}\right)$ which is subordinate to $\alpha$. Indeed, by Dowker's theorem there is a finite subcomplex $K$ of $X_{a \#}$ of dimension at most $p+1$ and a chain map which is subordinate to $\alpha^{\#}$,

$$
\pi: C_{*}\left(X_{a^{\#}}\right) \rightarrow C_{*}(K) .
$$

Since $K$ is finite there is a sequence of open coverings of $Y$,

$$
\gamma(-1)>\gamma(0)>\gamma(1)>\cdots>\gamma(p+1)>\gamma,
$$

such that, for any simplex $s \in K$, [St $\left.\mathrm{St}_{(i-1)}(s)\right]_{\gamma(i-1)}$ is p-acyclic in $\left[\mathrm{St}_{a(i)}(s)\right]_{\gamma(i)^{\circ}}$ Let $\alpha(i)=\alpha(p)$ and $\gamma(i)=\gamma(p)$ for $i \geq p$. Then define carriers $\Delta$ and $\Delta^{\prime}$ of $K$ into $C_{*}\left(Y_{\gamma}\right)$ by letting, for an $m$-simplex $s$ of $K$,

$$
\Delta(s)=C_{*}\left(\left[\mathrm{St}_{\alpha(m)}(s)\right]_{\gamma(m)}\right), \quad \Delta^{\prime}(s)=C_{*}\left(\left[\mathrm{St}_{\alpha_{(m-1)}}(s)\right]_{\gamma(m-1)}\right) .
$$

Then $\left(\Delta, \Delta^{\prime}\right)$ is a $p$-acyclic pair of interlaced carriers of $K$ into $C_{*}\left(Y_{\alpha}\right)$, and 2.1 provides a chain map which is carried by $\Delta$,

$$
\omega: C_{*}(K) \rightarrow C_{*}\left(Y_{\gamma}\right) .
$$

But then $\omega \pi$ is subordinate to $\alpha$.

4.2 Corollary. If $Y$ is a locally compact generalized cobomology manifold over $Q$, then $Y$ is a $Q$-simplicial space.

4.3 Corollary. If $Y$ is a locally compact generalized cobomology manifold over $Q$, then for any map $f: Y \rightarrow Y$ with a precompact image, the rank of $f$ is finite. If the Lefschetz number $\Lambda(f ; Q)$ is not zero, then $f(y)=y$ for some $y \in Y$.

Proof. Let $X$ be a compact subset of $Y$ which contains $f(Y)$. There is an open neighborhood $U$ of $X$ such that the inclusion map $j: U \rightarrow Y$ has finite rank. If $i: X \rightarrow U$ is the inclusion, then $f=j i\left(f_{X}\right)$. Then $f$ also has finite rank.

Now suppose that $\Lambda(f ; Q) \neq 0$. Let $g=i\left(f_{X}\right) j$. Then $\Lambda(g ; Q)=\Lambda(f ; Q)$. Furthermore $U$ is $Q$-simplicial at $X$ and $g_{X}=\left(f_{X}\right) j$ has finite rank. It follows from Theorem 1.1 that $g$ has a fixed point $y$, which is then a fixed point of $f$.

As a direct consequence of 4.1 there is

4.4 Theorem. If $Y$ is a topological manifold which is locally bomeomorphic to a topological vector space, then $Y$ is $Q$-simplicial at each of its subsets baving finite covering dimension. 
The techniques used to prove 4.3 are valid to prove

4.5 Corollary. If $Y$ is a topological manifold which is locally homeomorphic to a topological vector space, and $f$ is a map of $Y$ to itself sucb that the closure of $f(Y)$ is compact and of finite covering dimension, then the rank of $f$ is finite. If the Lefschetz number $\Lambda(f ; Q)$ is not zero, then $f$ bas a fixed point.

It is quite easy to construct a compact space $Y$ which is $\mathrm{lc}_{p}(Q)$, has covering dimension $p+1$, and has not finitely generated homology groups. For example, let $Y$ be the union of all the $p$-dimensional spheres in $R^{p+1}$ which are tangent to a hyperplane at a common point and which have radii $1 / 2^{n}$ for $n=1,2, \cdots$. Such a space can be neither a quasicomplex of Lefschetz nor a weak semicomplex of Thompson. By 4.1 such a space is $Q$-simplicial. However, there are compact spaces which are $l c_{p}(Q)$ and have covering dimension $p+2$ and are not $Q$-simplicial: Bing has given an example of a 2 -dimensional Peano continuum which has the Čech homology of a point, but which does not have the fixed point property [3]. It is therefore not $Q$-simplicial.

5. Fixed point theorems: The infinite dimensional case. The local connectivity hypothesis used in this section is the locally convexoid condition. It is much stronger than the $\mathrm{lc}_{p}(Q)$ condition, but more may be derived from it, and at the end of the section there will be given some applications to spaces constructed with attaching maps: cones, joins, and adjunction spaces.

5.1 Theorem. Suppose that $Y$ is a space and that $Y^{\prime}$ and $Y^{\prime \prime}$ are subsets such that $Y^{\prime}$ is relatively closed in $Y^{\prime} \cup Y^{\prime \prime}$. If $Y$ is locally convexoid at $Y^{\prime}$ and $Q$-simplicial at $Y^{\prime \prime}$, then $Y$ is $Q$-simplicial at $Y^{\prime} \cup Y^{\prime \prime}$.

Proof. Let $X$ be a compact subset of $Y$ and let $\alpha$ be an open covering of $X$. Suppose that $X$ is a subset of $Y^{\prime} \cup Y^{\prime \prime}$. We must find an open covering $a^{\#}$ of $X$ such that, for any open covering $\gamma$ of $Y$, there is a chain map of $C_{*}\left(X_{a^{\#}}\right)$ into $C_{*}\left(Y_{\gamma}\right)$ which is subordinate to $\alpha_{\text {. Let }} \alpha_{1}$ be an open covering of $X$ such that $\alpha_{1}^{*}>a$, let $X^{\prime}=X \cap Y^{\prime}$, and let $F$ be a monotone set function on $Y$, subordinate to $\alpha_{1}$ and such that some open covering $\alpha^{\prime}$ of $X^{\prime}$ is a gauge for $F$. Assume further that $\alpha^{\prime}>\alpha_{1}$. Let $\beta$ be an open covering of $X$ such that $\beta^{*}>\left\{A \backslash X^{\prime}: A \in \alpha\right\}$ $\cup \alpha^{\prime}$ and let $\beta^{\prime}=\left\{B \in \beta: B \cap X^{\prime} \neq \varnothing\right\}$. Let $V$ be an open neighborhood of $X \backslash$ st $_{\beta}\left(X^{\prime}\right)$ such that $\bar{V}$ is disjoint from $X^{\prime}$. Then $\bar{V} \cap X$ is a subset of $Y^{\prime \prime}$ and $Y$ is $Q$-simplicial at $\bar{V} \cap X$. Let $\beta^{\#}$ be an open covering of $\bar{V} \cap X$ such that for any open covering $\gamma$ of $Y$, there exists a chain map, subordinate to $\beta$,

$$
\omega^{\prime}: C_{*}\left([\bar{V} \cap X]_{\beta^{\#}}\right) \rightarrow C_{*}\left(Y_{\gamma}\right) \text {. }
$$

Let $\alpha^{\#}=\{V \cap B: B \in \beta\} \cup \beta^{\prime}$. Then it is claimed that, for any open covering 
$\gamma$ of $Y$, there exists a chain map, $\omega$, subordinate to $\alpha$, of $C_{*}\left(X_{a \#}\right)$ into $C_{*}\left(Y_{\gamma}\right)$. Indeed, since $\left(\alpha^{\#}\right)^{*}>\alpha_{1}^{*}>\alpha$, then by Dowker's theorem, it would suffice to define a chain map, subordinate to $\alpha_{1}$,

$$
\omega: C_{*}(K) \rightarrow C_{*}\left(Y_{\gamma}\right)
$$

for some finite $K$ in $X_{a_{\sharp}{ }^{*}}$. However, there does exist $\omega^{\prime}$ as above on $C_{*}\left([\bar{V} \cap X]_{\beta^{\sharp}}\right)$. So let $K^{\prime}=K \cap X_{\beta^{\prime}}$. Then $K$ is a subcomplex of $[\bar{V} \cap X]_{\beta^{\#}}$ $\cup K^{\prime}$. Let $L^{\prime}=K^{\prime} \cap[\bar{V} \cap X]_{\beta \sharp}$, and let $\omega^{\prime \prime}$ be the restriction of $\omega^{\prime}$ to $C_{*}\left(L^{\prime}\right)$. It suffices to extend $\omega^{\prime \prime}$ to a chain map $\omega^{\prime \prime \prime}$ of $C_{*}\left(K^{\prime}\right)$ into $C_{*}\left(Y_{\gamma}\right)$ which is subordinate to $\alpha_{1}$. This is to be done by an application of 2.1. For a simplex $s$ of $K^{\prime}$, diam St $\beta(s)<\beta^{*}$ and $\mathrm{St}_{\beta}(s) \cap X \neq \varnothing$. Then $\operatorname{diam} \mathrm{St}_{\beta}(s)<\alpha^{\prime}$, so $F\left(\mathrm{St}_{\beta}(s)\right)$ is $\infty$-acyclic. Let $p=\operatorname{dim} K^{\prime}$. There are only finitely many simplexes, so there exists a sequence of open coverings of $Y$,

$$
\gamma(-1)>\gamma(0)>\cdots>\gamma(p-1)>\gamma(p)=\gamma(p+1)=\cdots=\gamma
$$

such that, for $0 \leq m \leq p$ and $s \in K^{\prime},\left[F\left(\mathrm{St}_{\beta}(s)\right)\right]_{\gamma(m-1)}$ is acyclic in $\left[F\left(\mathrm{St}_{\beta}(s)\right)\right]_{\gamma(m)}$. So define carriers $\Delta, \Delta^{\prime}$ of $K^{\prime}$ to $C_{*}\left(Y_{\gamma}\right)$ by letting each be defined at an $m$-simplex $s$ of $K^{\prime}$ as

$$
\Delta(s)=C_{*}\left(\left[F\left(\mathrm{St}_{\beta}(s)\right)\right]_{\gamma(m)}\right), \quad \Delta^{\prime}(s)=C_{*}\left(\left[F\left(\mathrm{St}_{\beta}(s)\right)\right]_{\gamma(m-1)}\right) .
$$

Then $\left(\Delta, \Delta^{\prime}\right)$ is an $\infty$-acyclic pair of interlaced carriers of $K^{\prime}$ to $C_{*}\left(Y_{\gamma}\right)$. There is, by 2.1 , an extension of $\omega^{\prime \prime}$ to a chain map $\omega^{\prime \prime \prime}$ of $C_{*}\left(K^{\prime}\right)$ to $C_{*}\left(Y_{\gamma}\right)$ which is carried by $\Delta$. But $F \circ \mathrm{St}_{\beta}$ is subordinate to St $a_{1}$. Then $\omega^{\prime \prime \prime}$ is subordinate to $\alpha_{1}$, and if we define $\omega$ as $\omega^{\prime}$ on $C_{*}\left(K \cap[V \cap X]_{\alpha \#}\right)$ and as $\omega^{\prime \prime \prime}$ on $C_{*}\left(K^{\prime}\right)$, then $\omega$ is also subordinate to $\alpha_{1}$. This proves the theorem.

5.2 Corollary. Let $M$ be a topological manifold which is locally bomeomorphic to a locally convex space. If a map $f$ of $M$ into itself is sucb that the closure of $f(M)$ is compact, then $f$ has finite rank. If the Lefschetz number $\Lambda(f ; Q)$ is nonzero, then $f$ has a fixed point.

Proof. Let $X$ be the closure of $F(M)$. By 3.4 there is an open neighborhood $U$ of $X$ such that the inclusion $j: U \rightarrow Y$ has finite rank. Let $i: X \rightarrow U$ be the inclusion. Then $f=j i\left(f_{X}\right)$ has finite rank. If we let $g=i\left(f_{X}\right) j: U \rightarrow U$, then $\Lambda(f ; Q)=\Lambda(g ; Q)$, and $g_{X}=\left(f_{X}\right)$, which has finite rank. By 5.1,U is $Q$-simplicial at $X$, so if $\Lambda(g ; Q) \neq 0$, then $g$ has a fixed point, $x_{0}$. But then $x_{0}$ is a fixed point of $f$.

Suppose $Y$ is a space and $A$ is a closed subspace of $Y$. Let $T(Y, A)$ be the union $Y \times\{0\} \cup A \times[0,1]$. If $f: A \rightarrow Z$ is a map, let $Y \cup, Z$ be the space formed by attaching $Y$ to $Z$ by $f$; if $Y+Z$ is the disjoint topological sum of 
$Y$ and $Z$, let $p: Y+Z \rightarrow Y \cup, Z$ be the natural quotient map. For explicit definitions of these concepts, see [11]. Let $f_{1}: A \times\{1\} \rightarrow Z$ be defined by $f_{1}(a, 1)=f(a)$. Then $T(Y, A) \cup f_{1} Z$ is formed by attaching $T(Y, A)$ to $Z$ by $f_{1}$. The mapping cylinder $C(f)$ of $f$ is $C(f)=T(A, A) \cup_{f_{1}} Z$.

5.3 Theorem. If $Y$ is $Q$-simplicial, if $A \subset Y$ is a cofibration, and if $f: A \rightarrow Z$ is a map of $A$ to a locally convexoid space $Z$, then $Y \cup Z Z$ is a Q-simplicial space.

Proof. That $A \subset Y$ is a cofibration is equivalent to the existence of a retraction $\rho: Y \times[0,1] \rightarrow T(Y, A)$. By Corollary 3.11 of $[16], Y \times[0,1]$ is a $Q$-simplicial space and, by Corollary 3.5 of $[16], T(Y, A)$ is a $Q$-simplicial space. But then $T(Y, A) \cup f_{1} Z$ is $Q$-simplicial on the complement of $p(Z)$. Furthermore $T(Y, A) \cup_{f_{1}} Z$ is locally convexoid at $p(Z)$. By 5.1, $T(Y, A) \cup_{f_{1}} Z$ is a $Q$-simplicial space. The remainder of the proof consists in observing that since $A \subset Y$ is a cofibration, then, for any compact subset $X$ of $Y \cup, Z, X$ is approachable in $Y \cup, Z$ by $T(Y, A) \cup f_{1} Z$ and then, by Theorem 3.3 of [16], $Y \cup, Z$ is locally convexoid at $X$. Since $X$ was an arbitrary compact subset of $Y \cup, Z$, $Y \cup, Z$ is a $Q$-simplicial space.

5.4 Corollary. The mapping cylinder $C(f)$ of a map f from a $Q$-simplicial space to a locally convexoid space is Q-simplicial.

5.5 Corollary. The join $Y * Z$, of a $Q$-simplicial space $Y$ and a locally convexoid space $Z$, is a $Q$-simplicial space.

Proof. Let $p: Y \times Z \rightarrow Y, q: Y \times Z \rightarrow Z$ be the natural projections. Let $C(Z)$ be the cone over $Z$. Then $C(p) \simeq Y \times C(Z)$ so $C(p)$ is $Q$-simplicial. Furthermore $Y \times Z$ is identifiable to a closed subset of $C(p)$ such that $Y \times Z C$ $C(p)$ is a cofibration. Finally $Y * Z$ is formed by attaching $C(p)$ to $Z$ by $q$, so by $5.3, Y * Z$ is $Q$-simplicial.

5.6 Corollary. Let $Y$ be a $Q$-simplicial space and $C(Y)$ be the cone with base $Y$. Let $f$ be a map of $C(Y)$ to itself which has a precompact image. Then $f$ has a fixed point.

By a counterexample of Kinoshita, 5.6 is false for compact space $Y$ which is not $Q$-simplicial, and by an adjustment of Kinoshita's example, Knill showed that $C(Y)$ need not have the fixed point property even if $Y$ were compact and had the fixed point property itself. Thompson has shown, [24], that the cone over a compact quasi-complex has the fixed point property.

In general one cannot weaken the hypotheses of Theorem 5.3 by, say, weakening the condition on $Z$ to be $Q$-simplicial, or on $A$ to be any closed 
subset of $Y$. Example 4.1 of [16] may be used to construct a counterexample to the former, and as for the latter, it would suffice to see that

5.7 Theorem. There is a compact acyclic space $Y$ and closed subspace $A$ such that $A$ is locally convexoid and $Y$ is $Q$-simplicial at $Y \backslash A$, and a map $f: Y \rightarrow Y$ such that $f$ bas no fixed points.

Many of the known counterexamples of fixed point theory [5], [14], and [17], may be used to provide an example to prove 5.7.

\section{BIBLIOGRAPHY}

1. P. Alexandroff and H. Hopf, Topologie. I, Berlin, 1935. 734.

2. E. Begle, A fixed point theorem, Ann. of Math. (2) 51 (1950), 544-550. MR 11,

3. R. H. Bing, Challenging conjectures, Amer. Math. Monthly 74 (1967), no. 1, part II, 56-64. MR 34 \#3510.

4. A. Borel, The Poincaré duality in generalized manifolds, Michigan Math. J. 4 (1957), 227-239. MR 20 \#4842.

5. K. Borsuk, Sur un continu acyclique qui se laisse transformer topologiquement en lui meme sans points invarients, Fund. Math. 24 (1935), 51-58.

6. T. R. Brahana, Products of quasi-complexes, Proc. Amer. Math. Soc. 7 (1956), 954-958. MR 18, 328.

7. F. Browder, On the fixed point index for continuous mappings of locally connected spaces, Summa Brasil. Math. 4 (1960), 253-293. MR 26 \#4354.

8. - Fixed point theorems on infinite dimensional manifolds, Trans. Amer. Math. Soc. 119 (1965), 179-194. MR 33 \#3287. $1-25$.

9. E. Čech, Sur la connexité local d'ordre supérieur, Compositio Math. 2 (1935),

10. C. H. Dowker, Homology groups of relations, Ann. of Math. (2) 56 (1952), 84-95. MR 13,967 .

11. J. Dugund je, Topology, Allyn and Bacon, Boston, Mass., 1966. MR 33 \#1824.

12. J. Eells, A setting for global analysis, Bull. Amer. Math. Soc. 72 (1966), 751-807. MR $34 \# 3590$.

13. O. Hanner, Retraction and extension of mappings of metric and non-metric spaces, Ark. Mat. 2 (1952), 315-360. MR 14, 396; 1278.

14. S. Kinoshita, On some contractible continua without fixed point property, Fund. Math. 40 (1953), 96-98. MR 15, 642.

15. V. L. Klee, Leray-Schauder theory without local convexity, Math. Ann. 141 (1960), 286-296. MR 24 \#A1004.

16. R. J. Knill, Q-simplicial spaces, Illinois J. Math. 14 (1970), 40-51.

17. - Cones, products and fixed points, Fund. Math. 60 (1967), 35-46. MR $35 \# 2270$.

18. S. Lefschetz, Algebraic topology, Amer. Math. Soc. Colloq. Publ., vol. 27, Amer. Math. Soc., Providence, R. I., 1942. MR 4, 84.

19. J. Leray, Sur la forme des espaces topologiques et sur les points fixes des représentations, J. Math. Pures. Appl. (9) 24 (1945), 95-167. MR 7, 468.

20. - Théorie des points fixes: Indice total et nombre de Lefschetz, Bull. Soc. Math. France 87 (1959), 221-233. MR $26 \# 762$.

21. - La théorie des points fixes et ses applications en analyse, Proc. Internat. Congress Math. (Cambridge, Mass., 1950), vol. II, Amer. Math. Soc. Providence, R. I., 1952, pp. 202-208. MR 13,859. 
22. E. H. Spanier, Algebraic topology, McGraw-Hill, New York, 1966. MR 35 \#1007.

23. R. B. Thompson, On the semicomplexes of F. Browder, Bull. Amer. Math. Soc. 73 (1967), 531-536. MR 35 \#4902.

24. - A unified approach to local and global fixed point indices, Advances in Math. 3 (1969), 1-71. MR 40 \#891.

25. J. W. Tukey, Convergence and uniformity in topology, Ann. of Math. Studies, no. 2, Princeton Univ. Press, Princeton, N. J., 1940. MR 2, 67.

26. R. L. Wilder, Topology of manifolds, Amer. Math. Soc. Colloq. Publ., vol. 32, Amer. Math. Soc., Providence, R. I., 1949. MR 10, 614.

DEPARTMENT OF MATHEMATICS, TULANE UNIVERSITY, NEW ORLEANS, LOUISIANA 70118 